\section{Análise das temáticas publicadas pela Revista de Epidemiologia e Controle de Infecção}

Resumo: A fim de reconhecer as temáticas mais publicadas, entre 2011 a 2021, pela Revista de Epidemiologia e Controle de Infecção (RECI) realizou-se um estudo descritivo retrospectivo a partir de um levantamento e posterior análise de diversos assuntos com manuscritos já veiculados no periódico, com ênfase para o campo de doenças. Os resultados evidenciam que a maioria das publicações possuíam como temática a tuberculose (15\%), seguida das doenças crônicas não transmissíveis (13\%) e zoonoses (10\%). Observa-se que tais dados são necessários para identificar quais temáticas predominam, direcionando o enfoque do periódico, além de aperfeiçoá-lo e de definir estratégias, tais como chamadas para publicações especiais.

Palavras-chave: Temáticas publicadas. Publicações científicas. Análise Qualitativa.

Analysis of the themes published by the Journal of Epidemiology and Infection Control

Abstract: In order to recognize the most published themes, between 2011 and 2021, by the Journal of Epidemiology and Infection Control (JEIC), a retrospective descriptive study was carried out based on a survey and subsequent analysis of several subjects with manuscripts already published in the journal, with an emphasis on the field of diseases. The results show that most publications had tuberculosis as a theme $(15 \%)$, followed by non-communicable chronic diseases $(13 \%)$ and zoonoses $(10 \%)$. It is observed that such data are necessary to identify which themes predominate, directing the focus of the journal, in addition to improving it and defining strategies, such as calls for special publications.

Keywords: Published themes. Scientific publications. Qualitative analysis.

Análisis de los temas publicados por la Revista de Epidemiología y Control de Infecciones

Resumen: Con el fin de reconocer los temas más publicados, entre 2011 y 2021, por la Revista de Epidemiología y Control de Infecciones $(\mathrm{RECl})$, se realizó un estudio descriptivo retrospectivo a partir de una encuesta y posterior análisis de varios números con manuscritos ya publicados en la revista, con énfasis en el campo de las enfermedades. Los resultados muestran que la mayoría de las publicaciones tenían como tema la tuberculosis (15\%), seguida de las enfermedades crónicas no transmisibles (13\%) y las zoonosis (10\%). Se observa que dichos datos son necesarios para identificar qué temas predominan, orientar el enfoque de la revista, además de mejorarla y definir estrategias, como las convocatorias de publicaciones especiales.

Palabras Clave: Temas publicados. Publicaciones científicas. Análisis cualitativo.

\section{CASSIANDRA SAMPAIO JOAQUIM}

Santa Cruz do Sul, Rio

Grande do Sul, Brasil

Universidade de Santa

Cruz do Sul

\section{EZEQUIEL SERVEGNINI NUNES}

Santa Cruz do Sul, Rio

Grande do Sul, Brasil

Universidade de Santa

Cruz do Sul

\section{NATHALIA HALAX \\ ORFÃO}

Porto Velho, Rondônia,

Brasil

Universidade Federal de

Rondônia

\section{ANDREIA ROSANE DE MOURA VALIM}

Santa Cruz do Sul, Rio Grande do Sul, Brasil Universidade de Santa

Cruz do Sul

\section{LIA GONÇALVES POSSUELO}

Santa Cruz do Sul, Rio Grande do Sul, Brasil Universidade de Santa Cruz do Sul 


\section{Introdução}

A Revista de Epidemiologia e Controle de Infecção (RECl) é uma publicação oficial do Núcleo de Epidemiologia Hospitalar do Hospital Santa Cruz, de Santa Cruz do Sul/RS e do Programa de Pós-Graduação em Promoção da Saúde. Com periodicidade trimestral, tem por objetivo publicar artigos que contribuam para o conhecimento na área de epidemiologia e controle de infecção relacionados aos serviços de saúde. O corpo editorial é composto por profissionais de diversas instituições de ensino e com uma alta capacidade crítica e formadora de opinião.

Em maio de 2021, o periódico obteve um êxito importante, pois recebeu a certificação de indexação na conceituada base de dados Redalyc, que por sua vez, caracteriza a RECl como um dos "veículos" para a divulgação de experiências e estudos científicos relacionados aos assuntos de vigilância epidemiológica, assim como aos de controle de infecção relacionados às doenças de notificação compulsória, doenças infecciosas, saúde ocupacional e áreas correlatas. Além disso, na base de dados Web of Science, o periódico está classificado na categoria Doenças Infecciosas e Parasitárias.

Sob uma ótica geral, Imasato et al. (2017) destaca que artigos científicos publicados em periódicos indexados com revisão por pares têm recebido maior notoriedade e viabilizam, por exemplo, a estratificação das publicações, peculiarmente através de listas (ou rankings), que favorecem a classificação das revistas em consonância com o fator de impacto, percepção de qualidade ou prestígio do periódico.

Diante do exposto, o objetivo deste trabalho foi reconhecer o perfil das temáticas predominantemente publicadas pela RECl entre 2011 a 2021.

\section{Metodologia}

Trata-se de um estudo descritivo retrospectivo, cujos dados foram coletados no período de 23 de abril a 26 de maio de 2021, nos 39 números já publicados desde o primeiro número na RECl.

As temáticas foram elencadas a partir da observação do título do artigo, sendo que foram excluídos, as publicações dos suplementos das semanas acadêmicas do curso de Medicina publicadas anualmente no periódico. Os dados foram tabulados e analisados de forma descritiva utilizando o software Excel ${ }^{\mathrm{TM}}$. 


\section{Resultados}

Dos 39 números da RECI foram publicados 444 artigos, sendo a maioria artigos originais $(68,7 \%)$, seguido por revisão $(10,6 \%)$ e os demais comunicações breves e cartas ao editor. Foram identificadas 23 temáticas distintas, dentre elas sífilis, tuberculose (TB), HIV/Aids, hepatites, Covid-19, HPV, influenza, doenças crônicas não transmissíveis (DCNT), arboviroses, zoonoses, meningite, doenças parasitárias, hanseníase, coqueluche, varicela, doenças autoimunes, Síndrome Respiratória Aguda Grave (SRAG), sepse, Pneumonia Associada à ventilação mecânica (PAVM), cólera, micoses, síndrome de Fournier e neutropenia febril.

De modo complementar, verificou-se que as temáticas mais prevalentes foram a TB (15\%), DCNT (13\%), zoonoses (10\%), arboviroses (8\%), HIV/Aids (8\%) e sepse (7\%).

\section{Discussão}

A partir dos achados encontrados, as temáticas publicadas possuem relação com o escopo da revista e particularmente a TB também se caracteriza como um dos assuntos amplamente pesquisado e publicado pelo corpo editorial da $\mathrm{RECI}$, considerando que é a primeira entre as principais causas de morte por agente infeccioso, no mundo, superando o Vírus da Imunodeficiência Adquirida, causador da Síndrome da Imunodeficiência Adquirida (HIV/aids) (WHO, 2019), bem como trata-se da doença infectocontagiosa com maior coeficiente de incidência no Brasil (MINISTÉRIO DA SAÚDE, 2021), o qual também é um dos países prioritários tanto pela TB quanto a coinfecção TB/HIV (WHO, 2020), despertando interesse nos pesquisadores da área diante do grave e persistente problema de saúde pública.

Em relação as DCNT aparecem, principalmente, patologias como hipertensão, diabetes, doenças respiratórias crônicas, doenças cardiovasculares e neoplasias que possuem natureza multicausal e multifatorial, com longos períodos de latência e curso estendido, inclusive têm origem não infecciosa e podem refletir em incapacidades funcionais, corroborando para o recrudescimento dos custos econômicos e interferindo no desenvolvimento dos países (FIGUEIREDO et al., 2021). No Brasil, o Ministério da Saúde vem desenvolvendo várias ações em articulação com diversos setores governamentais e 
não governamentais objetivando promover a qualidade de vida, além de prevenir e controlar as DCNT.

Dentre as zoonoses, a raiva, leishmaniose e a toxoplasmose foram aquelas que apresentaram maior frequência nos estudos publicados na RECI. Segundo Ribeiro et al. (2020), tratam-se de infecções transmitidas dos animais às pessoas, seja de maneira direta por meio dos animais ou indireta através do consumo de alimentos contaminados e/ou por meio de vetores, perpassando a gravidade destas infecções no ser humano desde a ausência de sintomas até mesmo à morte.

Do mesmo modo, as arboviroses também se caracterizam como doenças transmitidas dos animais e insetos para o homem. Segundo o médico-veterinário Nélio Batista de Morais, presidente da Comissão Nacional de Saúde Pública Veterinária do Conselho Federal de Medicina Veterinária (2020): "De cada dez doenças que se apresentam clinicamente em seres humanos, pelo menos sete são causadas por agentes infecciosos pertencentes a gêneros que também já foram identificados em animais". Desta forma, a temática se torna (re)emergente, principalmente após a pandemia da Covid-19, considerando que o hospedeiro do Sars-Cov é um animal.

Considerada uma epidemia que teve início em 1981 e perpetua nos dias atuais, o HIV/Aids também se destacou dentre as temáticas, visto que sua importância perpetua até os dias atuais, por ser considerada a pior doença do século XX e não tem cura, apesar de ser tratável (BRITO, 2001).

Por fim, a temática sepse, as quais não são adquiridas apenas em hospitais, é a maior causa de morte nas Unidades de Terapia Intensiva, chegando a $65 \%$ dos casos no Brasil (PIFZER, 2019), reforçando sobre a importância dos cuidados em qualquer situação, sendo necessário ampliar as discussões acerca desta temática.

\section{Considerações finais}

As temáticas publicadas na RECl, em suas particularidades e especificidades, possuem importância para a sociedade e principalmente para o meio científico ao disseminar informação e conhecimento a partir de evidências científicas em favor da saúde pública e de ações coletivas de prevenção.

Para a RECI, este estudo mostra a importância que as temáticas têm e que precisam e merecem ganhar maior visibilidade na pesquisa científica. Desta forma e a partir desta 
análise, a revista fará chamadas abertas por temáticas com o objetivo de qualificar suas publicações no que tange ao escopo do periódico que a Epidemiologia e Controle de Infecção abarca.

\section{Referências}

BRASIL. Ministério da Saúde. Boletim Epidemiológico Tuberculose 2021. Disponível em: https://www.gov.br/saude/pt-br/media/pdt/2021/marco/24/boletimtuberculose2021 24.03\#: :text=A\%20tuberculose\%20(TB)\%20continua\%20sendo, milh\% C3\%A30\%20morreram\%20devido\%20\%C3\%A0\%20doen\%C3\%A7a. Acesso em 29 de junho de 2021

BRITO, Ana Maria de; CASTILHO, Euclides Ayres de; SZWARCWALD, Célia Landmann. AIDS e infecção pelo HIV no Brasil: uma epidemia multifacetada. Rev. Soc. Bras. Med. Trop. 2001; 34(2). Disponível em: https://doi.org/10.1590/S0037-86822001000200010. Acesso em 29 de junho de 2021

CFMV. Conselho Federal de Medicina Veterinária. Saúde Pública/Vigilância Epidemiológica: Tem sempre um médico-veterinário no combate às zoonoses e arboviroses. Disponível em: https://www.cfmv.gov.br/saude-publica-vigilanciaepidemiologica-tem-sempre-um-medico-veterinario-no-combate-as-zoonoses-earboviroses/comunicacao/noticias/2020/10/08/ Acesso em 29 de junho de 2021

FIGUEIREDO, Ana Elisa Bastos; CECCON, Roger Flores; FIGUEIREDO, José Henrique Cunha. Doenças crônicas não transmissíveis e suas implicações na vida de idosos dependentes. Ciên. Saúde Colet. 2021; 26(01). Disponível em: https://doi.org/10.1590/1413-81232020261.33882020 Acesso em: 29 jun 2021

IMASATO, Takeyoshi; PERLIN, Marcelo Scherer; BORENSTEIN, Denis. Análise do perfil dos acadêmicos e de suas publicações cientificas em Administração. Rev. adm. contemp. 2017; 21(1). Disponível em: https://doi.org/10.1590/1982-7849rac2017150329 Acesso em: 29 jun 2021

RIBEIRO, Ana Cristina Almeida; ARAÚJO, Rildo Vieira; ROSA, Andreza da Silva, et al. Zoonoses e Educação em Saúde: Conhecer, Compartilhar e Multiplicar. Braz. J. Hea. Rev. 2020; 3(5). Disponível em: https://doi.org/10.34119/bjhrv3n5-115. Acesso em: 29 jun 2021

WORLD HEALTH ORGANIZATION (WHO). Global Tuberculosis Report 2019. Geneva: WHO, 2019.

WORLD HEALTH ORGANIZATION (WHO). Global Tuberculosis Report 2020. Geneva: WHO, 2020. 
Cassiandra Sampaio Joaquim

Santa Cruz do Sul, Rio Grande do Sul, Brasil

Minicurrículo: Graduanda em Psicologia pela Universidade de Santa Cruz do Sul. Bolsista de Iniciação Científica da Revista de Epidemiologia e Controle de Infecção (RECl).

Contribuição de autoria: Escrita - Primeira Redação, Investigação, Metodologia.

ORCID ID: https://orcid.org/0000-0002-3987-4843

Lattes: http://lattes.cnpq.br/0344659666201071

E-mail: cassiandrajoaquim@gmail.com

\section{Ezequiel Servegnini Nunes}

Santa Cruz do Sul, Rio Grande do Sul, Brasil

Minicurrículo: Graduando em Enfermagem pela Universidade de Santa Cruz do Sul. Bolsista de Iniciação Científica da Revista de Epidemiologia e Controle de Infecção (RECI).

Contribuição de autoria: Escrita - Primeira Redação.

ORCID ID: https://orcid.org/0000-0002-7488-6890

Lattes: http://lattes.cnpq.br/9521123343257823

E-mail: ezequielnunes@mx2.unisc.br

\section{Nathalia Halax Orfão}

Porto Velho, Rondônia, Brasil

Minicurrículo: Doutorado pelo Programa de Pós-Graduação em Enfermagem em Saúde Pública da Escola de Enfermagem de Ribeirão Preto da Universidade de São Paulo. Professora da Fundação Universidade Federal de Rondônia (UNIR). Contribuição de autoria: Escrita - Revisão e Edição.

ORCID ID: https://orcid.org/0000-0002-8734-3393

Lattes: http://lattes.cnpq.br/7568632544062305

E-mail: nathaliahalax@unir.br

\section{Andreia Rosane de Moura Valim}

Santa Cruz do Sul, Rio Grande do Sul, Brasil

Minicurrículo: Doutorado em Biologia Celular e Molecular pela UFRGS. Professora adjunta da UNISC e docente do corpo permanente do Programa de Pós-Graduação em Promoção da Saúde da UNISC. Diretora de Inovação e Empreendedorismo da UNISC.

Contribuição de autoria: Escrita - Revisão e Edição.

ORCID ID: https://orcid.org/0000-0001-9611-3103

Lattes: http://lattes.cnpq.br/6480172642402703

E-mail: avalim@unisc.br 


\section{Lia Gonçalves Possuelo}

Santa Cruz do Sul, Rio Grande do Sul, Brasil

Minicurrículo: Doutorado em Ciências Biológicas (Bioquímica) pela UFRGS. Professora titular da UNISC e docente do corpo permanente do Programa de PósGraduação em Promoção da Saúde da UNISC. Editora chefe da Revista de Epidemiologia e Controle de Infecção (RECI).

Contribuição de autoria: Escrita - Revisão e Edição.

ORCID ID: https://orcid.org/0000-0002-6425-3678

Lattes: http://lattes.cnpq.br/9903194013924888

E-mail: liapossuelo@unisc.br

Esta obra está sob licença Creative Commons Atribuição 4.0 Internacional 